\title{
Profilaxia para tromboembolismo venoso em um hospital de ensino
}

\author{
Venous thromboembolism prophylaxis at a teaching hospital
}

\section{Rafael de M elo Franco ${ }^{1}$, Victor Simezo ${ }^{1}$, R afael Rodrigo Bortoleti ${ }^{1}$, Elias Lobo Braga ${ }^{1}$, Ana Rita Abrão ${ }^{1}$, Fábio Linardi ${ }^{2}$, José Augusto C osta ${ }^{3}$}

\section{Resumo}

O bjetivo: Verificar se a profilaxia da trombose venosa profunda está sendo utilizada de maneira correta e rotineira em um hospital de ensino.

M étodos e casuística: Foi realizado um estudo transversal de pacientes internados em sete setores (enfermarias) do Conjunto $\mathrm{H}$ ospitalar de Sorocaba (H ospital de Ensino), no período de agosto de 2004 a agosto de 2005. Para estratificação do risco de trombose venosa profunda de cada paciente, foram pesquisados fatores clínicos e cirúrgicos, segundo o protocolo preconizado pela SociedadeBrasileira deAngiologia e de Cirurgia Vascular. No período estudado, foram analisados 216 prontuários, dos quais 30 eram da cirurgia abdominal, 30 da cirurgia vascular, 30 da urologia, 31 da clínica médica, 31 da unidade de terapia intensiva, 31 da ortopedia e 33 da ginecologia/obstetrícia.

Resultados: Do total de pacientes, foi efetuada profilaxia para trombose venosa profunda em 57 (26\%), sendo que, em 51 (89\%), a execução foi de maneira correta e, em 6 (11\%), não-preconizada. 0 método profilático mais utilizado foi o medicamentoso; 49 de 57 pacientes fizeram uso de heparina de baixo peso molecular. Também foi verificada a utilização de meias elásticas em cinco pacientes e deambulação precoce em sete. Já a compressão pneumática intermitente não foi utilizada em nenhum deles.

C onclusão: D e acordo com os resultados e com base no protocoIo, concluiu-se que, no período da pesquisa, a profilaxia para trombose venosa profunda, no C onjunto H ospitalar de Sorocaba, foi executada rotineiramente e de forma adequada em apenas $23,6 \%$ (51 do total de 216 pacientes).

Palavras-chave: Trombose venosa profunda, profilaxia, tromboembolismo.

\begin{abstract}
O bjective: To verify whether deep venousthrombosis prophylaxis is being correctly and routinely used at a teaching hospital.

M ethods: A cross-sectional study of hospitalized patients on seven sectors at Conjunto H ospitalar de Sorocaba (H ospital de Ensino) was performed from August 2004 to August 2005. For the deep venous thrombosis risk stratification of each patient, clinical and surgical factors were investigated, according to the protocol recommended by Sociedade Brasileira de Angiologia e de C irurgia Vascular. D uring the period, 216 medical charts were analyzed. Of these, 30 were from abdominal surgery, 30 from vascular surgery, 30 from urology, 31 from medical clinic, 31 from intensive care unit, 31 from orthopedics and 33 from obstetrics/gynecology.
\end{abstract}

Results: 0 ut of the total number of patients, deep venous thrombosis prophylaxis was performed in $57(26 \%)$, considering that in $51(89 \%)$ the procedure was correct and in six (11\%) it did not follow the standard. The most used type of prophylaxis was drug treatment; 49 out of 57 patients used low-molecular-weight heparin. We also observed the use of elastic socks in five patients and early ambulation in seven. $0 \mathrm{n}$ the other hand, intermittent pneumatic compression was not used for any patient.

Conclusion: According to the results and based on the protocol, we concluded that, during the period of the research, deep venous thrombosis prophylaxis, at Conjunto Hospitalar de Sorocaba, was routinely and correctly performed in only $23.6 \%$ of the patients (51 out of 216).

Key words: D eep venous thrombosis, prophylaxis, thromboembolism.

1. Aluno de graduação, Curso de Medicina, Faculdade de Ciências Médicas, Centro de Ciências Médicas e Biológicas, Pontifícia Universidade Católica de São Paulo (CCMB/PUCSP), São Paulo, SP

2. Assistente Doutor, Departamento de Cirurgia, Faculdade de Ciências Médicas, CCMB/PUCSP, São Paulo, SP.

3. Professor titular, Departamento de Cirurgia, Faculdade de Ciências Médicas, CCMB/PUC-SP, São Paulo, SP

O presente estudo foi apresentado no XXII Congresso da SUMEP, no VII Congresso Paulista Médico-Acadêmico, no V Encontro Local de Iniciação Científica e no XIV Encontro de Iniciação Científica da PUCSP.

Auxílio: O presente trabalho foi realizado como programa de iniciação científica, com aprovação e apoio do Conselho Nacional de Desenvolvimento Científico e Tecnológico (CNPq).

Artigo submetido em 27.03.06, aceito em 09.06.06. 
A trombose venosa profunda (TVP) é uma doença de ocorrência multidisciplinar, caracterizada pela formação de trombos de forma oclusiva total ou parcial, em veias do sistema venoso profundo, efreqüentemente relacionada a diversos fatores de riscos, que podem promover seu aparecimento súbito em pacientes hígidos ou como complicação clínica e/ou cirúrgical-5.

A TVP éaterceiracausa mortisdedoença cardiovascular nos EUA, ocorrendo, anualmente, em $1 \%$ da população ${ }^{2}$. A incidência da doença teve um leve decréscimo nas últimas décadas, porém, o tromboembolismo pulmonar (TEP) e a TVP ainda constituem um importanteproblema desaúde pública ${ }^{3-5}$. N o H ospital das C línicas da Faculdade de M edicina de Botucatu, 0 TEP apareceu em 19,1\% das necropsias, sendo a causa do óbito em 3,7\% desses pacientes. Além disso, a insuficiênciavenosacrônica, como síndromepós-trombótica, apesar de não-mortal, constitui um problema socioeconômico importante 5 .

U mavariedadedefatores predispõeàT VP, estando estes diretamente relacionados à tríade de Virchow (estasevenosa, discrasia sangüínea ealteraçõesno endotélio). 0 reconhecimento desses fatores é necessário tanto para o diagnóstico quanto para o estabel ecimento do risco trombótico potencial e, assim, do regime profilático mais adequado ${ }^{1,3,5}$.

Algumaspesquisas mostraram uma baixa aderência à profilaxia contra os eventos trombóticos ou seu uso incorreto, quando realizada. N o entanto, a prevenção adequada reduz em doisterços os casos deT V P eem um terço os casos de TEP, o que torna extremamente importante 0 conhecimento dos diferentes grupos de risco e seus meios de prevenção $1,3,5$.

\section{0 bjetivos}

Verificar sea profilaxia daTVP está sen do utilizada de maneira preconizada e rotineira no Conjunto $\mathrm{H}$ ospitalar de Sorocaba (CH S).

\section{M aterial e método}

Foi realizado um estudo transversal de pacientes internados, abordando as especialidades clínica médi$\mathrm{Ca}$, ortopedia, cirurgia geral (urologia, cirurgia vascular e abdominal), ginecologia/ obstetrícia (GO ) e unidade de terapia intensiva (UTI). 0 s pacientes foram divididos em duas classes: pacientes internados para tratamento clínico e para tratamento cirúrgico, sendo paci- ente cirúrgico todo aquele que fora submetido a al gum procedimento cirúrgico na atual internação. $\mathrm{N}$ ão houve a aplicação de um termo de consentimento livre e esclarecido, pois não ocorreram entrevistas nem contato direto com os pacientes. As análises foram realizadas através do exame do prontuário médico, sendo que a identificação dos pacientes foi totalmente preservada durante a verificação desses prontuários. 0 período da pesquisa foi de 12 meses.

Foi analisado um total de 216 prontuários de pacientes, 111 do sexo masculino e 105 do feminino, com média deidade de 49,98 anos (4-90), internados no C H S, nas enfermarias de clínica médica, ortopedia, urologia, cirurgia vascular, cirurgia abdominal, GO e UTI.

Deste montante, $31(14,35 \%)$ eram da clínica médica, $31(14,35 \%)$ da ortopedia, $30(13,88 \%)$ da urologia, 30 (13,88\%) dacirurgiavascular, $30(13,88 \%)$ da cirurgia abdominal, $33(15,27 \%)$ da GO e 31 $(14,35 \%)$ da UTI, mostrado na Tabela 1.

Para estratificação do risco de TVP de cada paciente, foram pesquisados fatores clínicos, medicamentosos e cirúrgicos, segundo o protocolo preconizado pela Sociedade Brasileira de Angiologia e Cirurgia V ascular (SBACV) ${ }^{6}$ (Anexo 1) e Caprini ${ }^{7}$. A correta utilização da profilaxia foi analisada segundo os critérios da SBACV6, que estabelece uma classificação de riscos (alto, médio ebaixo) através de pontuações.

Tabela 1 - Clínicas pesquisadas e realização da profilaxiarelação entre os setores abordados e a execução ou não de profilaxia para TVP

\begin{tabular}{lccc}
\hline Setores & Executou & Não executou & T otal \\
\hline Cirurgia abdominal & 4 & 26 & 30 \\
Cirurgia vascular & 15 & 15 & 30 \\
U rologia & 8 & 22 & 30 \\
Clínica médica & 6 & 25 & 31 \\
UTI & 11 & 20 & 31 \\
Ortopedia & 12 & 19 & 31 \\
GO & 1 & 32 & 33 \\
Total & 57 & 159 & 216 \\
\hline
\end{tabular}

GO = ginecologia/obstetrícia; TVP = trombose venosa profunda; UTI = unidade de terapia intensiva. 
A partir disso, o protocolo recomendaumaconduta através das diversas modalidades de profilaxia, como: deambulação precoce, contensão elástica, compressão pneumática intermitente e medicamentosa (heparina não-fracionada e heparina de baixo peso molecular). Portanto, considerou-se uso inadequado da profilaxia os casos em que, estabel ecida a pontuação pela análise dos prontuários, a conduta realizada não estava de acordo com a recomendada (Anexo 1).

N os prontuários analisados, não havia nenhuma descrição de sinais ou sintomasque pudessem suspeitar da presença de TVP ou TEP, considerados critérios de exclusão para o estudo.

\section{Resultados}

N o setor da clínica médica, foram analisados 31 prontuários, sendo 10 deles classificados como de al to risco, 18 de médio risco e três de baixo risco. Em seis pacientes, dosquaisum era dealto risco ecinco eram de médio risco, foi realizada alguma forma de profilaxia para TVP. Em quatro, foram executadas medidas preventivas, de acordo com o protocolo preconizado pela SBACV , e, em dois, foi utilizada apenas deambulação precoce em pacientes com indicação para medicamentos. $\mathrm{N}$ os outros 25 prontuários avaliados, não foi encontrado nenhum tipo deprofilaxia. $D$ entreesses, nove eram de al to risco, 13 de médio e três de baixo risco.

$\mathrm{N}$ o setor da ortopedia, foram analisados 31 prontuários, sendo 28 deles classificados como de alto risco, dois de médio risco e um de baixo risco. Em 12 pacientes, foi realizada alguma forma de profilaxia preconizada pela SBACV, sendo que 11 eram de alto risco e um de médio risco. $\mathrm{N}$ os outros 19 prontuários avaliados, não foi encontrado nenhum tipo de profilaxia. D entre esses, 17 eram de al to risco, um de médio e outro de baixo risco.

$\mathrm{N}$ o setor da urologia, foram analisados 30 prontuários, sendo 14 del esclassificadoscomo deal to risco, 15 de médio risco e um de baixo risco. Em oito pacientes, foi realizada profilaxia, sendo sete de forma medicamentosa. D os que executaram, cinco eram de al to risco e três de médio risco. Nos outros 22 prontuários avaliados, não foi encontrado nenhum tipo de profilaxia, apesar de terem indicação, segundo critérios da SBACV. D entre esses, nove eram de alto risco, 12 de médio e um de baixo risco.

N o setor da cirurgia vascular, dos 30 prontuários, 18 deles foram classificados como de alto risco e
12 de médio risco. Em 15 pacientes, foi realizada profilaxia, sendo 12 com medicamentos. Dos que realizaram a profilaxia, 10 eram de alto risco e cinco de médio risco. N os outros 15 prontuários aval iados, não foi encontrado nenhum tipo de profilaxia, apesar da indicação. $D$ entre esses, oito eram de al to risco e sete de médio.

N o setor da cirurgia abdominal, foram analisados 30 prontuários, sendo 16 deles classificados como de alto risco, 11 de médio risco e três de baixo risco. Em quatro pacientes, foi realizada al guma forma de profilaxia paraT V P. N osoutros 26 prontuáriosavaliados, não foi encontrado nenhum tipo de profilaxia, apesar de terem indicação, segundo critérios da SBACV. D entre esses, 15 eram de al to risco, 10 de médio e um de baixo risco.

N o setor da GO , foram analisados 33 prontuários, sendo cinco del es classificados como de al to risco, 18 de médio risco e 10 de baixo risco. Em apenas um paciente, classificado como médio risco, foi realizada profilaxia. N os outros 32 prontuários aval iados, não foi encontrado nenhum tipo de profilaxia, apesar de terem indicação, segundo critérios da SBACV . D entre esses, cinco eram de alto risco, 17 de médio e 10 de baixo risco.

N o setor da U TI, foram anal isados 31 prontuários, sendo 11 deles classificados como de alto risco, 18 de médio risco e dois de baixo risco. Em 11 pacientes, foi realizada corretamenteal guma forma de profilaxia para TVP. D os que executaram, seis eram de alto risco e cinco de médio risco. $\mathrm{N}$ os outros 20 prontuários avaliados, não foi encontrado nenhum tipo de profilaxia. D entre esses, cinco eram de alto risco, 13 de médio e dois de baixo risco.

O s pacientes que não receberam profilaxia representaram um total de 151 , ou seja, $73,6 \%$. D esses pacientes, $73(33,8 \%)$ eram de médio risco, sendo a maioria dentre os grupos de risco. Já o grupo de pacientes de alto risco era de 68 (31,5\%).

Os tipos de profilaxia pesquisados foram: meia elástica, compressão pneumática intermitente, deambulação precoce e medicamentos (T abela 2).

D e todos os tipos de profilaxia pesquisados, o mais utilizado foi o medicamentoso, sendo efetuado em 49 pacientes. 0 setor que mais empregou a profilaxia foi a cirurgia vascular, e a forma medicamentosa foi a mais usada. Jáa compressão pneumática intermitentenão foi utilizada em nenhum dos pacientes estudados. 
T abela 2 - C línicas pesquisadas e meios de prevenção - relação entre os setores abordados e os tipos de profilaxia utilizados

\begin{tabular}{lccccc}
\hline Setores & $\begin{array}{c}\text { Sem } \\
\text { profilaxia }\end{array}$ & $\begin{array}{c}\text { M eias } \\
\text { elásticas }\end{array}$ & $\begin{array}{c}\text { Compressão } \\
\text { pneumática } \\
\text { intermitente }\end{array}$ & $\begin{array}{c}\text { Deambulação } \\
\text { precoce }\end{array}$ & Medicamento \\
\hline Cirurgia abdominal & 26 & 2 & 0 & 2 & 2 \\
Cirurgia vascular & 15 & 3 & 0 & 2 & 12 \\
U rologia & 22 & 0 & 0 & 1 & 7 \\
Clínica médica & 25 & 0 & 0 & 2 & 4 \\
UTI & 20 & 0 & 0 & 0 & 11 \\
Ortopedia & 19 & 0 & 0 & 0 & 12 \\
GO & 32 & 0 & 0 & 0 & 1 \\
\hline
\end{tabular}

$\mathrm{GO}=$ ginecologia/obstetrícia; $\mathrm{UTI}$ = unidade de terapia intensiva .

\section{D iscussão}

A prevenção da TVP é fundamental e necessária para se evitar suas complicações agudas, como o TEP, ou crônicas, como a síndrome pós-trombótica. Principalmente pela natureza muitas vezes silenciosa da TVP, a embolia pulmonar pode ser sua primeira manifestação ${ }^{8}$.

Pelas evidências constatadasedadosconsistentesna literatura, as medidas profiláticas são cada vez mais discutidas e indicadas, juntamente com 0 aprimoramento dos protocolos ${ }^{9}$.

Segundo alguns consensos, como o de $\mathrm{N}$ icolaides, sobre a freqüência de TVP sem profilaxia, é clara a necessidade da sua utilização, como a taxa de $25 \%$ em cirurgia geral, $51 \%$ em cirurgia el etiva de quadril, $50 \%$ no trauma, $47 \%$ em cirurgia do joelho, $45 \%$ em fratura do quadril, $56 \%$ no acidentevascular cerebral e22\% em neurocirurgia ${ }^{10}$.

A análise dos dados obtidos, no $\mathrm{CH}$ S, evidencia que a grande maioria dos pacientes, 159 (74\%), atendidos nas respectivas clínicas, não estava recebendo métodos profiláticos para TV $P$, o que demonstra que, embora acessíveis, estes são pouco utilizados. M esmo em países considerados desenvolvidos, como no estudo publicado por Goldhaber \& Tapson, no qual, dos 2.726 pacientes com diagnóstico de TVP, apenas 42\% (1.147 pacientes) havia recebido profilaxia, destaca-se também essa consideração.

Em nosso meio, os dados são parecidos com essa subutilização da profilaxia, conformeal guns estudosna literatura. Engelhorn ${ }^{3}$, em um estudo epidemiológico em hospital-escola, evidenciou que $87,28 \%$ dos 228 pacientes não receberam tratamento profilático para TVP. Caiafa \& Bastos ${ }^{11} 2$ encontraram $65,9 \%$ sem profilaxia, sendo $40,01 \%$ de forma inadequada. M archi et al. ${ }^{12}$, em uma avaliação de um hospital geral, encontraram $87,39 \%$ dos pacientes sem profilaxia e $62,9 \%$ de maneira inadequada.

Ficam algumas dúvidas e questionamentos quanto a essa baixa aplicação de métodos preventivos para TVP, já que está bem documentada sua incidência.

Seria o tipo de classificação de grupos de risco? 0 receio da ocorrência de sangramentos em pacientes cirúrgicos, embora tenha sido mostrado que 0 uso das substâncias profiláticas não aumentou o seu risco ${ }^{13}$ 0 u porque os especial istas desconhecem a real incidência de TVP e/ou TEP em seus pacientes?

Considerando a execução da profilaxia por setor estudado, constatou-se que a cirurgia vascular, com 15 pacientes, $50 \%$ dosestudadosnestesetor, foi o quemais executou profilaxia: $6,94 \%$ do total. No entanto, 0 setor que menos executou foi o $\mathrm{GO}$, com um paciente, $3 \%$ dos estudadosno setor e $0,46 \%$ do total (T abela 3 ). 
T abela 3 - G rupos derisco ea prevenção deT VP - relação entre a classificação de risco ea execução ou não de profilaxia para TVP

\begin{tabular}{|c|c|c|c|c|c|c|c|}
\hline \multirow[t]{2}{*}{ Setores } & \multicolumn{2}{|c|}{ B aixo risco } & \multicolumn{2}{|c|}{ M édio risco } & \multicolumn{2}{|c|}{ Alto risco } & \multirow[t]{2}{*}{ T ota } \\
\hline & Executou & $\mathbf{N}$ ão executou & Executou & Não executou & Executou & $\mathbf{N}$ ão executou & \\
\hline Cirurgia abdominal & 2 & 1 & 1 & 10 & 1 & 15 & 30 \\
\hline Cirurgia vascular & 0 & 0 & 5 & 7 & 10 & 8 & 30 \\
\hline U rologia & 0 & 1 & 3 & 12 & 5 & 9 & 30 \\
\hline Clínica médica & 0 & 3 & 5 & 13 & 1 & 9 & 31 \\
\hline UTI & 0 & 2 & 5 & 13 & 6 & 5 & 31 \\
\hline O rtopedia & 0 & 1 & 1 & 1 & 11 & 17 & 31 \\
\hline GO & 0 & 10 & 1 & 17 & 0 & 5 & 33 \\
\hline T otal & 2 & 18 & 21 & 73 & 34 & 68 & 216 \\
\hline
\end{tabular}

GO = ginecologia/obstetrícia; TVP = trombose venosa profunda; UTI = unidade de terapia intensiva.

$\mathrm{N}$ os casos em que a profilaxia foi realizada, ou seja, em 57 pacientes $(26 \%)$, ela foi indicada e realizada corretamente em $23,6 \%$ (51 pacientes) e, em seis $(2,4 \%)$, não estava de acordo com os critérios da SBACV (T abela 4).

\section{Conclusão}

D e acordo com os resultados e com base no proto- colo de profilaxia de TVP, montado sob as diretrizes da SBACV, concluiu-se que, no período da pesquisa, a profilaxiadeT VP nestehospital deensino foi executada rotineiramente e de forma adequada em $23,6 \%$ (51 do total de 216 pacientes) e em $2,4 \%$ dos pacientes de maneira incorreta (seis do total de 216).

Constatou-se que a profilaxia não foi realizada em 159 pacientes (74\%).

T abela 4 - Análise da execução de profilaxia - número de pacientes tratados, de acordo com os critérios da SBACV, e não tratados

\begin{tabular}{lccc}
\hline \multirow{2}{*}{ Setores } & \multicolumn{2}{c}{ Execução da profilaxia } & \multirow{2}{*}{ T otal } \\
\cline { 2 - 3 } & Preconizada & $\mathbf{N}$ ão-preconizada & \\
\hline Cirurgia abdominal & 4 & 0 & 4 \\
Cirurgia vascular & 12 & 3 & 15 \\
U rologia & 7 & 1 & 8 \\
Clínica médica & 4 & 2 & 6 \\
UTI & 11 & 0 & 11 \\
O rtopedia & 12 & 0 & 12 \\
GO & 1 & 0 & 1 \\
Total & 51 & 6 & 57 \\
\end{tabular}

GO = ginecologia/obstetrícia; SBACV = Sociedade Brasileira de Angiologia e Cirurgia Vascular; UTI = unidade de terapia intensiva. 


\section{Anexo 1}

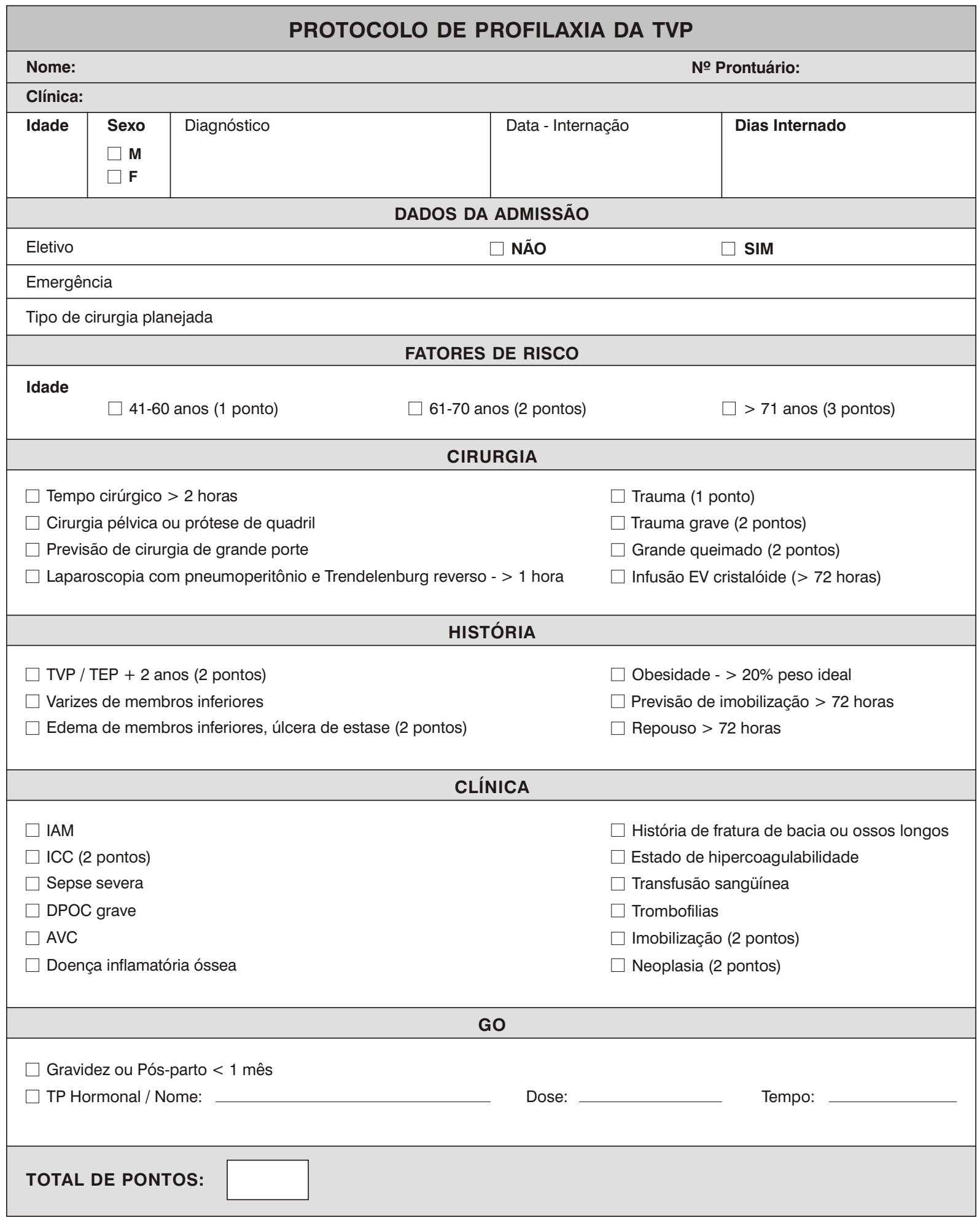




\section{Anexo 1}

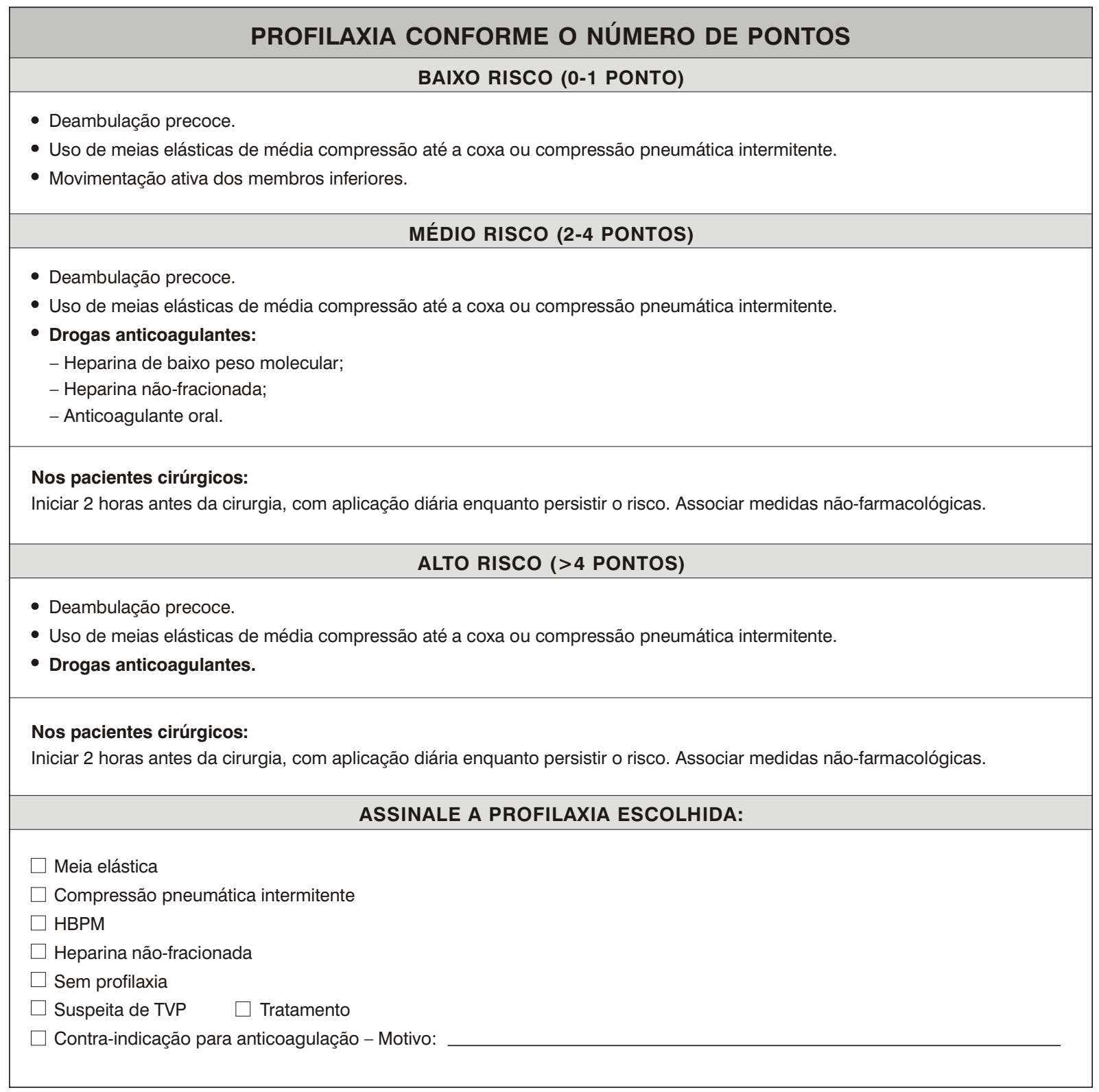

\section{Referências}

1. Anand SS, W ells PS, H unt D, Brill-Edwards P, Cook D, Ginsberg JS. Does this patient have deep vein thrombosis? JAM A. 1998;14:1094-9.

2. D ryjski $M, O$ 'B rien-I rr M S, $H$ arris LM , H assett J, J anickeD . Evaluation of screening protocol to exclude the diagnosis of deep venous thrombosis among emergency department patients. J V asc Surg. 2001;34:1010-5.
3. Engelhorn CA, Garcia ACF, Cassou MF, Birckholz L, Engelhorn ALV. Profilaxia da trombose venosa profunda estudo epidemiológico em um hospital escola. J Vasc Bras. 2002;1:97-102.

4. H ollyoak M , W oodruff P, M uller M , D aunt N, W eir P. D eep venous thrombosis in postoperative vascular surgical patients: a frequent finding without prophylaxis. J V asc Surg. 2001;34: 656-60. 
5. Maffei $F H A$, Rollo HA. Trombose venosa profunda dos membros inferiores: incidência, patologia, patogenia, fisiopatologia e diagnóstico. In: M affei FHA, Lastódia S, YoshidaW B, Rollo H A. D oenças vasculares periféricas. 3a ed. Rio de Janeiro: M ED SI. 2002. p. 1363-86.

6. N ormas deorientação clínica para a prevenção diagnóstica eo tratamento da trombose venosa profunda. J Vasc Bras. 2005;4(Supl. 3):S205-20.

7. Caprini JA, Arcelus I, Reyna JJ. Effectiverisk stratification of surgical and nonsurgical patientsfor venous thromboembolic disease. Semin Hematol. 2001;38(2 Suppl 5):12-9.

8. Baker WF Jr. Diagnosis of deep venous thrombosis and pulmonary embolism. M ed C lin N orth Am. 1998;82:459-76.

9. G eerts WH, H eit JA, Clagett $G P$, et al. Prevention of venous thromboembolism. Chest. 2001;119:132S-75S.

10. N icolaides AN, Breddin HK, Fareed J, et al. Prevention of venous thromboembolism: International Consensus Statement. Guidelines compiled in accordance with the scientific evidence. J V asc Bras. 2002;1:133-70.
11. Caiafa JS, Bastos M. Programa de profilaxia do tromboembolismo venoso do $\mathrm{H}$ ospital $\mathrm{N}$ aval $\mathrm{M}$ arcilio $\mathrm{D}$ ias: um modelo de educação continuada. J Vasc Bras. 2002;1: 103-12.

12. M archi C, Schlup IB, Lima CA, Schlup HA. Avaliação da profilaxia da trombose venosa profunda em um H ospital Geral. J Vasc Bras. 2005;4:171-5.

13. Jorgensen $L N, W$ ille J orgensen $P, H$ auch $O$. Prophylaxis of postoperative thromboembolism with low molecular weight heparins. Br J Surg. 1993;80:689-704.

\section{Correspondência:}

José Augusto Costa

Av. W ashington Luis, 845

CEP 18030-270 - Sorocaba, SP

Tel.: (15) 3233.2833

E-mail: drcosta@globo.com

O conteúdo do J Vasc Bras está disponível em português e em inglês

no site do Jornal Vascular Brasileiro em

www.jvascbr.com.br 